\title{
Equação de Burgers em um Domínio Arbitrário
}

\author{
L.A. FIOREZE, Departamento de Matemática-CEUNIFRAN, Santa Maria, RS.
}

J.P. LUKASZCZYK, Departamento de Matemática-UFSM, Santa Maria, RS.

Resumo. Neste trabalho provamos a existência e unicidade de soluções fracas para a equação vetorial de Burgers em domínios arbitrários em três dimensões. A única hipótese considerada sobre o domínio é que este seja um aberto. As estimativas para estes resultados utilizam uma desigualdade de Sobolev do tipo elíptica apresentada nos preliminares.

\section{Introdução}

O sistema de equações de Burgers pode ser interpretado como uma simplificação do sistema de Navier-Stokes onde não consideramos a força devido ao gradiente de pressão e a condição de incompressibilidade do fluído, isto é,

$$
\left\{\begin{array}{l}
u_{t}+u \cdot \nabla u-\mu \Delta u=f \text { em } \Omega \times(0, T), \\
u(x, t)=0 ; \quad x \in \partial \Omega ; \forall t \in(0, T), \\
u(x, 0)=u_{0}(x) .
\end{array}\right.
$$

Aqui, $u(x, t)=\left(u_{1}(x, t), u_{2}(x, t), u_{3}(x, t)\right), \Delta u=\left(\Delta u_{1}, \Delta u_{2}, \Delta u_{3}\right)$ e $u \cdot \nabla u=$ $\sum_{i=1}^{3} u_{i} \frac{\partial u}{\partial x_{i}}, \quad \mu$ é uma constante (considerada igual a 1 ) e $f$ um campo de força externo dado. Tal sistema de equações, foi primeiramente tratado por J. M. Burgers num artigo de 1948 (veja Burgers [3]) onde se estudou algumas questões relativas a turbulência. Neste trabalho, utilizando o método de Galerkin, apresentamos resultados de existência e unicidade de soluções fracas em espaços de Sobolev para a equação de Burgers, com $f \equiv 0$ em domínios arbitrários. Uma referência básica utilizada é Heywood [6] onde se mostra a existência de soluções $C^{\infty}((0, T) \times \Omega)$ para um $T$ adequado. Aqui seguimos passos semelhantes aos feitos no artigo de Heywood sendo que a descrição do método de Galerkin, o que não é desenvolvido no artigo de Heywood, é apresentada neste trabalho.

Além disto, utilizando um lema que fornece estimativas em $\widehat{H}_{0}^{1}(\Omega)$ que é um espaço que contém o $H_{0}^{1}(\Omega)$ (veja lemas 1 e 2), obtemos outros resultados de limitação para a solução.

O trabalho está dividido em três seções principais; na primeira são apresentados alguns preliminares básicos para o estudo de soluções fracas de equações diferenciais parciais assim como o Lema 2. Na seção seguinte apresentamos resultados 
de existência de solução fraca para o sistema de equações de Burgers em domínios limitados. Na terceira seção apresentamos resultados de existência de solução em domínios não limitados. Como é usual em problemas envolvendo equações diferenciais parciais, $C$ denotará uma constante dependendo somente dos dados do problema e poderá variar de linha para linha.

\section{Preliminares}

No desenvolvimento desta seção, apresentaremos algumas definições e resultados básicos utilizados no estudo das equações diferenciais parciais, assim como alguns resultados mais específicos que se tornaram necessários no estudo da equação a que nos propomos.

Proposição 1 (Desigualdade de Gronwall). Seja $\eta(t)$ uma função absolutamente contínua e não-negativa em $[0, T]$ e que satisfaz quase sempre a inequação diferencial $\eta^{\prime}(t) \leq \phi(t) \eta(t)+\psi(t)$ onde $\phi(t)$ e $\psi(t)$ são funções integráveis nãonegativas em $(0, T)$. Então

$$
\eta(t) \leq e^{\int_{0}^{t} \phi(s) d s}\left[\eta(0)+\int_{0}^{t} \psi(s) d s\right] \quad \text { para } 0 \leq t \leq T .
$$

Demonstração. Veja Evans [5] na página 556.

Observação 1. (operador $-\Delta$ )

O operador $-\Delta$ desempenha um papel importante na teoria das equações diferenciais parciais pois suas autofunções constituem uma base ideal em certos espaços funcionais. O principal resultado referente a este operador afirma que cada autovalor é real com multiplicidade finita (número de vezes que o autovalor se repete) e forma uma seqüência $0<\lambda_{1} \leq \lambda_{2} \leq \lambda_{3} \leq \ldots$, onde $\lambda_{k} \rightarrow \infty$, quando $k \rightarrow \infty$ e que existe uma base ortonormal $\left\{w_{k}\right\}_{k \in \mathbb{N}}$ de $H_{0}^{1}(\Omega)$, onde $\Omega$ é um aberto limitado. Assim $-\Delta w_{k}=\lambda_{k} w_{k}$ e $\left.w_{k}\right|_{\partial \Omega} \equiv 0$. Para mais detalhes e demonstrações veja Evans [5] na página 301.

Teorema 1. Sejam $X_{0}, X, X_{1}$ espaços de Hilbert onde $X_{0} \subset X \subset X_{1}$, com injeções contínuas sendo que a injeção de $X_{0}$ em $X$ é compacta. Então, para qualquer $K$ limitado e dado $\gamma>0$, a injeção de $\mathbb{H}_{K}^{\gamma}\left(\mathbb{R} ; X_{0}, X_{1}\right)$ em $L^{2}(\mathbb{R}, X)$ é compacta onde

$$
\begin{aligned}
& \mathbb{H}^{\gamma}\left(\mathbb{R} ; X_{0}, X_{1}\right)=\left\{v \in L^{2}\left(\mathbb{R}, X_{0}\right) ; D_{t}^{\gamma} v \in L^{2}\left(\mathbb{R}, X_{1}\right)\right\}, \\
& \mathbb{H}_{k}^{\gamma}\left(\mathbb{R} ; X_{0}, X_{1}\right)=\left\{u \in \mathbb{H}^{\gamma}\left(\mathbb{R} ; X_{0}, X_{1}\right): \operatorname{supp} u \subset K\right\} .
\end{aligned}
$$

Demonstração. Veja Temam [9] na página 274.

Lema 1. Dado $\Omega \subset \mathbb{R}^{3}$, um aberto. Seja $\left(\Omega_{n}\right)_{n \in \mathbb{N}}$ uma seqüência de subdomínios limitados tais que $\Omega_{1} \subset \Omega_{2} \subset \Omega_{3} \subset \ldots$ e $\Omega=\bigcup_{n=1}^{\infty} \Omega_{n}$ e seja $u_{0} \in \widehat{H}_{0}^{1}(\Omega)$ (fecho de $C_{0}^{\infty}(\Omega)$ na norma de $|\nabla \phi|$, isto é, $\widehat{H}_{0}^{1}(\Omega)=\left\{\phi \in L_{\text {loc }}^{1}(\Omega)\right.$ com $\nabla \phi \in$ $L^{2}(\Omega): \exists \phi_{n} \in C_{0}^{\infty}(\Omega)$ com $\left|\nabla\left(\phi_{n}-\phi\right)\right| \rightarrow 0$ quando $\left.\left.n \rightarrow \infty\right\}\right)$. Então existe uma seqüência de funções $u_{n} \in H_{0}^{1}\left(\Omega_{n}\right)$ com $\operatorname{supp} u_{n} \subset \bar{\Omega}_{n}$ e $\left|\nabla u_{n}\right| \leq\left|\nabla u_{0}\right|$ e tais que $\left|\nabla u_{n}-\nabla u_{0}\right| \rightarrow 0$ quando $n \rightarrow \infty$. 
Demonstração. Veja Heywood [7] na página 665.

Lema 2. Suponha que $\Omega$ é um aberto em $\mathbb{R}^{3}$. Então, dada $u \in \widehat{H}_{0}^{1}(\Omega)$ com $\Delta u \in$ $L^{2}(\Omega)$, tem-se:

$$
|u|_{\infty} \leq \frac{1}{\sqrt{2 \pi}}|\nabla u|^{\frac{1}{2}}|\Delta u|^{\frac{1}{2}}
$$

Demonstração. Veja Xie [10].

\section{Equação de Burgers em um Domínio Limitado}

Nesta seção mostraremos a existência da aproximação de Galerkin em dimensão finita, faremos algumas estimativas a priori para a Equação de Burgers e finalmente mostraremos existência e unicidade de solução em um domínio limitado. Aqui, supomos que $\Omega$ é um domínio limitado em $\mathbb{R}^{3}$.

O teorema abaixo fornece condições para a existência e unicidade de solução fraca para a Equação de Burgers em um domínio tridimensional limitado $\Omega \subset \mathbb{R}^{3}$.

Teorema 2 (Existência e Unicidade de Solução). Suponha que $\Omega$ é um domínio limitado em $\mathbb{R}^{3}$ e que $u_{0} \in H_{0}^{1}(\Omega)$. Seja $T=\frac{256 \pi^{2}}{27\left|\nabla u_{0}\right|^{4}}$ então existe uma única solução $u \in L^{2}\left(0, T ; H_{0}^{1}(\Omega)\right)$ satisfazendo

$$
\frac{\partial u}{\partial t}+u \cdot \nabla u=\Delta u \text { em } H^{-1}(\Omega) \text { e no sentido das distribuições } D^{\prime}(0, T) .
$$

Além disto, $\lim _{t \rightarrow 0^{+}}\left|\nabla u(t)-\nabla u_{0}\right|=0$ e $|\nabla u(t)|^{2} \leq \frac{\left|\nabla u_{0}\right|^{2}}{\sqrt{1-\frac{t}{T}}}, \quad 0 \leq t<T$.

A demonstração deste teorema é desenvolvida nas quatro subseções a seguir.

\subsection{Existência de Solução em Dimensão Finita}

Nesta subseção mostraremos a formulação variacional para a equação:

$$
u_{t}+u \cdot \nabla u=\Delta u, \quad u(0)=u_{0} \text { e }\left.u\right|_{\partial \Omega}=0
$$

e a existência de solução para a formulação variacional em dimensão finita.

Aplicaremos o método de Galerkin.

Seja $\left\{\phi_{1}, \phi_{2}, \phi_{3}, \ldots\right\}$ uma base ortonormal de $L^{2}(\Omega)$, consistindo de autofunções do Laplaciano: $\Delta \phi_{n}=-\lambda_{n} \phi_{n} ; \phi_{n} \in H_{0}^{1}(\Omega)$.

Para cada $n$ define-se uma solução aproximada $u_{n}(x, t)=\sum_{i=1}^{n} C_{n, i}(t) \phi_{i}(x)$

A formulação variacional no espaço $V_{n}=\left\{\phi_{1}, \phi_{2}, \ldots \phi_{n}\right\}$ é

$$
\frac{d}{d t}\left(u_{n}, \phi_{j}\right)-\left(\Delta u_{n}, \phi_{j}\right)+\left(u_{n} \cdot \nabla u_{n}, \phi_{j}\right)=0, \forall \phi_{j} \in H_{0}^{1}(\Omega),
$$




$$
u_{n}(x, 0)=u_{0 n}, \quad \forall j=1,2, \ldots, n
$$

onde $u_{0 n}$ é a projeção ortogonal de $u_{0}$ no espaço $V_{n}$, isto é, o espaço gerado por $\left\{\phi_{1}, \phi_{2}, \phi_{3}, \ldots, \phi_{n}\right\}$.

Isto é equivalente a um sistema de equações diferenciais ordinárias de primeira ordem nos coeficientes $C_{1}(t), C_{2}(t), \ldots, C_{n}(t)$ (observe que devido ao fato da base de autofunções ser ortogonal em $L^{2}$ e $H_{0}^{1}$ os coeficientes $C$ não dependem da dimensão $n$ ). Tal sistema juntamente com a condição inicial (3.2) define, devido ao Teorema de existência e unicidade de solução de equações diferenciais ordinárias, uma única solução (veja Coddington \& Levison [4]). A solução obtida é local no tempo sendo que as estimativas a priori permitirão prolongar para $t \in(0, T)$.

\subsection{Estimativas a Priori}

Seja $u_{n}$ a n-ésima aproximação de Galerkin e, por conveniência, não usaremos o subscrito $n$. Fazendo o produto interno em $L^{2}(\Omega)$ da equação por $v=-\Delta u$ (o que é possível por causa da Observação 1 pois $\left.\Delta u \in H_{0}^{1}\right)$, obtemos

$$
\frac{1}{2} \frac{d}{d t}|\nabla u|^{2}+|\Delta u|^{2}=(u \cdot \nabla u, \Delta u) .
$$

Desta equação, utilizando Desigualdade de Young, Lema de Gronwall e Lema 2 obtemos as seguintes estimativas

$$
\begin{aligned}
& |\nabla u|^{2} \leq \frac{\left|\nabla u_{0}\right|^{2}}{\sqrt{1-\frac{t}{T}}}, \int_{0}^{t}|u|_{\infty}^{2} d s \leq b_{1}(t), \int_{0}^{t}|\Delta u|^{2} d s \leq \frac{\left|\nabla u_{0}\right|^{2}}{2\left(1-\sqrt[6]{\frac{t}{T}}\right) \sqrt{1-\sqrt{\frac{t}{T}}}}, \\
& \int_{0}^{t}\left|u_{t}\right|^{2} d s \leq b_{2}(t) \quad \text { e }\left|u(t)-u_{0}\right| \leq \sqrt{b_{2}(t)} \sqrt{t}
\end{aligned}
$$

onde as limitantes $b_{1}(t)$ e $b_{2}(t)$ são funções contínuas em $\left(t,\left|\nabla u_{0}\right|\right) \in[0, T) \times[0, \infty)$, as quais são independentes do subíndice $n$ e $\Omega$ além disto $T$ é o número definido no Teorema 2 .

\subsection{Existência de Solução em um Domínio Limitado}

Integrando em $t$ a primeira estimativa em (3.4), tem-se

$$
\int_{0}^{T}|\nabla u(t)|^{2} d t \leq \int_{0}^{T} \frac{\left|\nabla u_{0}\right|^{2}}{\sqrt{1-\frac{t}{T}}} d t=\lim _{\varepsilon \rightarrow T^{+}} \int_{0}^{\varepsilon} \frac{\left|\nabla u_{0}\right|^{2}}{\sqrt{1-\frac{t}{T}}} d t=2 T\left|\nabla u_{0}\right|^{2}
$$

Assim, $u \in L^{2}\left(0, T ; H_{0}^{1}(\Omega)\right)$.

No sentido de passarmos o limite com $n \rightarrow \infty$ na formulação de Galerkin (3.1) precisamos de uma convergência forte (por causa do termo $u \cdot \nabla u$ ) do tipo

$$
u_{n} \longrightarrow u \text { em } L^{2}\left(0, T ; L^{2}(\Omega)\right) .
$$


Neste sentido vamos utilizar uma idéia semelhante a desenvolvida por Temam [9] onde foi utilizada uma derivada fracionária. Infelizmente não podemos utilizar derivada comum pois $\lim _{t \rightarrow T} \int_{0}^{t}\left|u_{t}\right|^{2} d s=\infty$, e assim não podemos garantir a limitação de $u_{t}$ em $L^{2}(\Omega)$. Mas $D_{t}^{\gamma} u$ pode ser definida para um $\gamma$ adequado como veremos a seguir. Seja $\widetilde{u}_{n}$ uma função de $\mathbb{R}$ em $H_{0}^{1}(\Omega)$ que é igual a $u_{n}$ em $[0, T]$ e zero no complemento deste intervalo, denotemos a transformada de Fourier de $\widetilde{u}_{n}$ é denotada por $\widehat{u}_{n}$, quer-se mostrar que

$$
\int_{-\infty}^{\infty}|r|^{2 \gamma}\left|\widehat{u}_{n}(r)\right|^{2} d r \leq \text { constante }
$$

para algum $\gamma>0$ pois isto implica que $\widetilde{u}_{n} \in \mathbb{H}^{\gamma}\left(\mathbb{R}, H_{0}^{1}, L^{2}\right)$.

Para demonstrar (3.5) primeiramente observemos que (3.1) pode ser reescrita como:

$$
\frac{d}{d t}\left(\widetilde{u}_{n}, \phi_{j}\right)=\left(\Delta \widetilde{u}_{n}, \phi_{j}\right)-\left(\widetilde{u}_{n} \cdot \nabla \widetilde{u}_{n}, \phi_{j}\right)+\left(u_{0 n}, \phi_{j}\right) \delta_{0}-\left(u_{n}(T), \phi_{j}\right) \delta_{T},
$$

para $j=1,2, \ldots, n$, sendo $\delta_{0}$ e $\delta_{T}$ distribuições de Dirac, onde os funcionais $\delta_{0}$ e $\delta_{T}$ em $D(\mathbb{R})$ são dados por $\delta_{0}: C_{0}^{\infty} \longrightarrow \mathbb{R}$ onde $\left\langle\delta_{0}, \varphi\right\rangle=\varphi(0)$ e $\delta_{T}: C_{0}^{\infty} \longrightarrow \mathbb{R}$ onde $\left\langle\delta_{T}, \varphi\right\rangle=\varphi(T)$.

Aplicando a transformada de Fourier em (3.6), obtém-se

$$
2 i \pi r\left(\widehat{\widetilde{u}}_{n}, \phi_{j}\right)=\left\langle\widehat{f}_{n}, \phi_{j}\right\rangle+\left(u_{0 n}, \phi_{j}\right)-\left(u_{n}(T), \phi_{j}\right) e^{-2 i \pi r T}
$$

sendo $\widehat{\widetilde{u}}_{n}$ e $\widehat{\widetilde{f}}_{n}$ a transformada de Fourier de $\widetilde{u}_{n}$ e $\widetilde{f}_{n}$, respectivamente e $\widetilde{f}_{n}=$ $\left(\Delta u_{n} ; \phi_{j}\right)-\left(u_{n} \cdot \nabla u_{n} ; \phi_{j}\right)$ sendo definida como zero fora do intervalo $(0, T)$.

Multiplicando a equação $(3.7)$ por $\widehat{C}_{n, j}(t)$ (o qual denota a transformada de Fourier de $C_{n, j}$ que é o coeficiente da aproximação de Galerkin em dimensão finita) e adicionando as equações resultantes para $j=1,2, \ldots, n$, obtém-se

$$
2 i \pi r\left|\widehat{u}_{n}(r)\right|^{2}=\left\langle\widehat{f}_{n}, \widehat{u}_{n}\right\rangle+\left(u_{0 n}, \widehat{u}_{n}\right)-\left(u_{n}(T), \widehat{u}_{n}\right) e^{-2 i \pi r T} .
$$

Tem-se,

$$
\begin{aligned}
\int_{0}^{T}\left|f_{n}\right|_{H^{-1}} d t \leq\left(\int_{0}^{T} d t\right)^{\frac{1}{2}}\left(\int_{0}^{T}\left|u_{n}\right|_{H_{0}^{1}}^{2} d t\right)^{\frac{1}{2}}+\int_{0}^{T}\left|u_{n}\right|_{H_{0}^{1}}^{2} d t \\
=\sqrt{T}\left(\int_{0}^{T}\left|u_{n}\right|_{H_{0}^{1}}^{2} d t\right)^{\frac{1}{2}}+\int_{0}^{T}\left|u_{n}\right|_{H_{0}^{1}}^{2} d t \leq C
\end{aligned}
$$

pois $u_{n}$ é limitado em $L^{2}\left(0, T ; H_{0}^{1}\right)$.

Como $f_{n} \in L^{1}\left(0, T ; H^{-1}\right)$, então $\widehat{f}_{n} \in L^{\infty}\left(0, T ; H^{-1}\right)$, pois

$$
\left|\widehat{f}_{n}(t)\right|_{H^{-1}} \leq C \int_{-\infty}^{\infty}\left|e^{-2 i \pi r t}\right||f(r)|_{H^{-1}} d r=C \int_{-\infty}^{\infty}|f(r)|_{H^{-1}} d r
$$


Tem-se então:

$$
\int_{-\infty}^{\infty}|r|^{2 \gamma}\left|\widehat{u}_{n}(r)\right|^{2} d r \leq C_{3} \int_{-\infty}^{\infty}\left|\widehat{u}_{n}(r)\right|^{2} d r+C_{4} \int_{-\infty}^{\infty} \frac{\left|\widehat{u}_{n}(r)\right|_{H_{0}^{1}}}{1+|r|^{1-2 \gamma}} d r
$$

Assim temos que $D_{t}^{\gamma} \widetilde{u}_{n} \in L^{2}\left(\mathbb{R}, L^{2}(\Omega)\right)$ para $0<\gamma<\frac{1}{4}$. Portanto, podemos utilizar o Teorema 1 e concluir que, para uma subsequência,

$$
u_{n^{\prime}} \rightarrow u \quad \text { em } L^{2}\left(0, T ; L^{2}\right) \text { fortemente. }
$$

No sentido de passar o limite em $n$ na equação (3.1) seja agora $\psi(t) \in C^{\prime}[0, T]$, com $\psi(T)=0$. Multiplicando (3.1) por $\psi$ e integrando de 0 a $T$, obtém-se

$-\int_{0}^{T}\left(u_{n}, \psi^{\prime} v\right) d t+\int_{0}^{T}\left(u_{n} \cdot \nabla u_{n}, \psi v\right) d t+\int_{0}^{T}\left(\nabla u_{n}, \nabla v \psi\right) d t=\left(u_{n}(0), \psi(0) \cdot v\right)$

Portanto, no limite, tem-se:

$$
-\int_{0}^{T}(u, v) \psi^{\prime} d t+\int_{0}^{T}(u \cdot \nabla u, v) \psi d t+\int_{0}^{T}(\nabla u, \nabla v) \psi d t=\left(u_{0}, v\right) \psi(0)
$$

Finalmente, prova-se que $u$ satisfaz $u(0)=u_{0}$. Para isto, da formulação variacional, multiplica-se a equação

$$
\frac{d}{d t}(u, v)+(u \cdot \nabla u, v)=(\Delta u, v), \quad \forall v \in H_{0}^{1}
$$

por $\psi(t)$ e integra-se de 0 a $T$. Após, integrando-se por partes e por comparação com $(3.10)$, observa-se que $\left(u_{0}-u(0), v\right) \psi(0)=0$ para cada $v$ em $H_{0}^{1}$ e para cada $\psi$ como definido anteriormente. Escolhe-se $\psi$ tal que $\psi(0) \neq 0$. Portanto, $\left(u(0)-u_{0}, v\right)=0, \quad \forall v \in V$ o que implica que $u(0)=u_{0}$.

No sentido de mostrar que $\nabla u(t) \rightarrow \nabla u_{0}$ fortemente quando $t \rightarrow 0^{+}$, primeiramente mostramos que $\nabla u(t) \rightarrow \nabla u_{0}$ fracamente em $H_{0}^{1}(\Omega)$, isto é, para cada $\phi_{i} \in C_{0}^{\infty}(\Omega)$, fixo, temos que o termo

$$
\int_{\Omega}\left(\nabla u(t)-\nabla u_{0}\right) \nabla \phi_{i}(x) d x \rightarrow 0
$$

quando $t \rightarrow 0^{+}$

Mas, como $|\nabla u(t)|^{2} \leq \frac{\left|\nabla u_{0}\right|^{2}}{\sqrt{1-\frac{t}{T}}}$ e fazendo $t \rightarrow 0^{+}$, tem-se $\lim _{t \rightarrow 0^{+}}|\nabla u|^{2} \leq\left|\nabla u_{0}\right|^{2}$ e, assim, tem-se que $\nabla u(t) \rightarrow \nabla u_{0}$ quando $t \rightarrow 0^{+}$fortemente. 


\subsection{Unicidade de Solução}

Suponha que $u$ e $v$ sejam soluções da equação de Burgers no sentido do Teorema 2. Assim,

$$
\left\{\begin{array} { l } 
{ u _ { t } - \Delta u + u \cdot \nabla u = 0 } \\
{ u ( 0 ) = u _ { 0 } ; u | _ { \partial \Omega } \equiv 0 }
\end{array} \quad \left\{\begin{array}{l}
v_{t}-\Delta v+v \cdot \nabla v=0 \\
v(0)=u_{0} ;\left.v\right|_{\partial \Omega} \equiv 0
\end{array}\right.\right.
$$

em $H^{-1}(\Omega)$

Seja $w=u-v$ e

$$
\left\{\begin{array}{l}
w_{t}-\Delta w+u \cdot \nabla u-v \cdot \nabla v=0 \\
w(0)=0
\end{array} .\right.
$$

Tem-se que

$$
u \cdot \nabla u-v \cdot \nabla v=v \cdot \nabla w+w \cdot \nabla u
$$

Substituindo (3.13) em (3.12) e fazendo o produto interno com $\Delta w$ em $L^{2}$, tem-se

$$
\left(w_{t}, \Delta w\right)+(v \cdot \nabla w, \Delta w)+(w \cdot \nabla u, \Delta w)=(\Delta w, \Delta w) .
$$

Então,

$$
\frac{1}{2} \frac{d}{d t}|\nabla w|^{2}+|\Delta w|^{2}=(v \cdot \nabla w, \Delta w)+(w \cdot \nabla u, \Delta w) .
$$

Usando as Desigualdades de Cauchy-Schwarz, Young e Hölder,

$(v \cdot \nabla w, \Delta w)+(w \cdot \nabla u, \Delta w) \leq C_{\varepsilon}|v|_{\infty}^{2}|\nabla w|^{2}+\varepsilon|\Delta w|^{2}+C_{\varepsilon}|\nabla w|^{2}|\nabla u|^{4}+\varepsilon|\Delta w|^{2}$.

Usando a limitação dada acima em (3.14) e escolhendo $\varepsilon=1$, obtém-se

$$
\frac{d}{d t}|\nabla w|^{2} \leq C|\nabla w|^{2}\left(|v|_{\infty}^{2}+|\nabla u|^{4}\right)
$$

Devido à Desigualdade de Gronwall,

$$
|\nabla w(t)|^{2} \leq \exp \left[C\left(|v|_{\infty}^{2}+|\nabla u|^{4}\right)\left(t-t_{1}\right)\right]\left|\nabla w\left(t_{1}\right)\right|^{2}
$$

Fazendo $t_{1} \rightarrow 0^{+}$

$$
|\nabla w(t)|^{2} \leq \exp \left[C\left(|v|_{\infty}^{2}+|\nabla u|^{4}\right) t\right] \cdot 0=0
$$

o que implica que $w(t)=0$, ou seja, a solução é única.

\section{A Equação de Burgers em um Domínio não Limitado}

Nesta seção vamos demonstrar a seguinte versão do Teorema 2 para a equação de Burgers em um domínio $\Omega$ não limitado. 
Teorema 3 (Existência e Unicidade de Solução). Suponha que $\Omega$ é um domínio não limitado em $\mathbb{R}^{3}$ e que $u_{0} \in \widehat{H}_{0}^{1}(\Omega)$. Seja $T=\frac{256 \pi^{2}}{27\left|\nabla u_{0}\right|^{4}}$ então existe uma única solução u com $u(t) \in \widehat{H}_{0}^{1}(\Omega)$ satisfazendo:

$$
\begin{gathered}
\frac{\partial u}{\partial t}+u \cdot \nabla u=\Delta u, \text { em } H^{-1}(\Omega) \text { e no sentido das distribuições } D^{\prime}(0, T), \\
\lim _{t \rightarrow 0^{+}}\left|\nabla u(t)-\nabla u_{0}\right|=0 \text { e }|\nabla u(t)|^{2} \leq \frac{\left|\nabla u_{0}\right|^{2}}{\sqrt{1-\frac{t}{T}}}, \quad 0 \leq t<T .
\end{gathered}
$$

Demonstração. Devido ao Lema 1 , dado $u_{0} \in \widehat{H}_{0}^{1}(\Omega)$, é possível escolher $u_{n, 0} \in$ $C_{0}^{\infty}(\Omega)$ tais que $\left|\nabla u_{n, 0}-\nabla u_{0}\right| \rightarrow 0$ quando $n \rightarrow \infty ;\left|\nabla u_{n, 0}\right| \leq\left|\nabla u_{0}\right|$. Escolhemos uma seqüência de conjuntos abertos limitados $\left\{\Omega_{m}\right\}$ tais que $\Omega_{1} \subset \Omega_{2} \subset \Omega_{3} \subset \ldots$ e $\Omega=\bigcup_{m=1}^{\infty} \Omega_{m} \operatorname{com} \operatorname{supp} u_{m, 0} \subset \bar{\Omega}_{m}$.

De acordo com o Teorema 2 , em cada $\Omega_{m}$ com dado inicial $u_{m, 0}$ encontramos uma solução $u_{m}$ da equação de Burgers. Definimos: $\bar{u}_{m}=u_{m}$ em $\Omega_{m}$ e $\bar{u}_{m}=0$ em $\left.\Omega\right|_{\Omega_{m}}$

Observe que $\bar{u}_{m}$ é a solução da Equação de Burgers em $\Omega$ com dado inicial $u_{m, 0}$ definido como zero fora de $\Omega_{m}$.

Tem-se

$$
\frac{\partial \bar{u}_{m}}{\partial t}+\bar{u}_{m} \cdot \nabla \bar{u}_{m}=\Delta \bar{u}_{m} \quad \text { onde } \quad \bar{u}_{m}(t) \in \widehat{H}_{0}^{1}(\Omega) ; t \in(0, T)
$$

com

$$
\lim _{t \rightarrow 0^{+}}\left|\nabla \bar{u}_{m}(t)-\nabla u_{m, 0}\right|=0 \text { e }\left|\nabla \bar{u}_{m}(t)\right|^{2} \leq \frac{\left|\nabla u_{m, 0}\right|^{2}}{\sqrt{1-\frac{t}{T}}} .
$$

Vamos construir uma seqüência da seguinte forma.

Em $\Omega_{1}$ consideremos uma subseqüência de $\left(\bar{u}_{n}\right)_{n \in \mathbb{N}}$ denotada por $w_{1}^{1}, w_{2}^{1}, w_{3}^{1}, \ldots$ que converge fraco em $L^{2}\left(0, T ; H_{0}^{1}\left(\Omega_{1}\right)\right) \cap L^{\infty}\left(0, T ; L^{2}\left(\Omega_{1}\right)\right)$.

Em $\Omega_{2}$ consideramos uma subseqüência de $\left(w_{n}^{1}\right)_{n \in \mathbb{N}}$ denotada por $w_{1}^{2}, w_{2}^{2}, w_{3}^{2}, \ldots$ que converge fraco em $L^{2}\left(0, T ; H_{0}^{1}\left(\Omega_{2}\right)\right) \cap L^{\infty}\left(0, T ; L^{2}\left(\Omega_{2}\right)\right)$.

Em geral, em $\Omega_{m}$ consideremos uma subseqüência de $\left(w_{n}^{m-1}\right)_{n \in \mathbb{N}}$ denotada por $w_{1}^{m}, w_{2}^{m}, w_{3}^{m}, \ldots$ que converge fraco em $L^{2}\left(0, T ; H_{0}^{1}\left(\Omega_{m}\right)\right) \cap L^{\infty}\left(0, T ; L^{2}\left(\Omega_{m}\right)\right)$.

A seqüência $\left(w_{1}^{1}, w_{2}^{2}, w_{3}^{3}, \ldots\right)$ que simplificadamente denotamos por $\left(w_{1}, w_{2}, \ldots\right)$ tem a propriedade de que para qualquer $\Omega_{l}$ dado e $\forall T^{\prime}<T$ convergir fracamente para $w$ em $L^{2}\left(0, T^{\prime} ; H_{0}^{1}\left(\Omega_{l}\right)\right)$.

Além disso, o limite $w$ satisfaz

$$
\lim _{t \rightarrow 0^{+}}\left|\nabla w(t)-\nabla u_{0}\right|=0 \text { e }|\nabla w(t)|^{2} \leq \frac{\left|\nabla u_{0}\right|^{2}}{\sqrt{1-\frac{t}{T}}} .
$$


No sentido de obtermos uma solução fraca, considere $\phi \in C_{0}^{\infty}\left(\Omega_{m}\right)$; $\phi \in C_{0}^{1}\left(\Omega_{m} \times[0, T]\right)$ e definindo $\phi \equiv 0$ em $\Omega \backslash \Omega_{m}$, tem-se:

$$
\int_{0}^{T^{\prime}} \int_{\Omega}\left(\frac{d}{d t} w_{n}+w_{n} \cdot \nabla w_{n}-\Delta w_{n}\right) \phi d x d t=0
$$

$\forall n \geq m$ e $0<T^{\prime}<T$.

Passando o limite com $n \rightarrow \infty$ em (4.17), obtemos que

$$
\int_{0}^{T^{\prime}} \int_{\Omega}\left(\frac{d}{d t} w_{n}+w_{n} \cdot \nabla w_{n}-\Delta w_{n}\right) \phi d x d t \rightarrow \int_{0}^{T^{\prime}} \int_{\Omega}\left(\frac{d}{d t} w+w \cdot \nabla w-\Delta w\right) \phi d x d t .
$$

Assim,

$$
\int_{0}^{T^{\prime}} \int_{\Omega}\left(\frac{d}{d t} w+w \cdot \nabla w-\Delta w\right) \phi d x d t=0
$$

$\forall \phi \in C_{0}^{\infty}\left(\Omega_{m}\right), \phi \in C_{0}^{1}\left(\Omega_{m} \times[0, T]\right)$.

Portanto,

$$
\frac{d}{d t} w+w \cdot \nabla w-\Delta w=0
$$

em $H^{-1}(\Omega)$ e no sentido das distribuições $D^{\prime}(0, T)$.

A prova para a condição inicial $\left|\nabla w(t)-\nabla u_{0}\right| \rightarrow 0$ quando $t \rightarrow 0^{+}$para domínios não limitados é feita de forma semelhante ao caso de domínio limitado, com pequenas diferenças, ou seja, como $|\nabla w(t)|^{2} \leq \frac{\left|\nabla u_{0}\right|^{2}}{\sqrt{1-\frac{t}{T}}}$ então $\lim _{t \rightarrow 0^{+}} \sup |\nabla w(t)|^{2} \leq$

$\left|\nabla u_{0}\right|^{2}$. Assim para mostrar que $\nabla w(t) \rightarrow \nabla u_{0}$ fortemente em $L^{2}(\Omega)$ quando $t \rightarrow$ $0^{+}$precisamos mostrar que $\nabla w(t) \rightarrow \nabla u_{0}$ fracamente em $L^{2}(\Omega)$. A demonstração é totalmente similar a feita para o caso limitado, sendo que a diferença reside em que $\int_{\Omega} \nabla\left(w_{n}(0)-u_{0}\right) \cdot \nabla a_{i} d x \rightarrow 0$ quando $n \rightarrow \infty$.

Agradecimentos: Os autores agradecem ao professor Luis Adauto Medeiros pelas críticas e sugestões recebidas.

Abstract. In this work we prove the existence and uniqueness of weak solutions of the vector Burgers equation in arbitrary three dimensional domains. The assumption about the spatial domain is that it should be an open set. The underlying estimates for these results are proved using a elliptic-Sobolev type inequality present at the preliminaries.

\section{Referências}

[1] R. Adams, "Sobolev Spaces", Academic Press, New York, 1975.

[2] H. Brezis, "Analyse Fonctionnelle", 2a Tiragem, Masson S. A., 1987. 
[3] J.M. Burgers, A mathematical model illustrating the theory of turbulence, Adv. Appl. Math., 1 (1948), 171-199.

[4] E.A. Coddington e N. Levinson, "Theory of Ordinary Differential Equations", McGraw Hill, 1955.

[5] L.C. Evans, "Partial Differential Equations", Berkeley Mathematics Lectures Notes, V. 3A, Berkeley, Ca, 1993.

[6] J.B. Heywood e W. Xie, Smoth solution of the vector Burgers equation in nonsmooth domains, Differential and Integral Equations, 10, No. 5 (1997), 961-974.

[7] J.B. Heywood, The Navier-Stokes equations: on the existence, regularity and decay of solutions, Indiana University Mathematics Journal, 29, No. 5 (1980), 639-681.

[8] L.A. Medeiros e E.A. Milla, "A Integral de Lebesgue - Texto Didático", Série Matemática / 1, Editora Universitária, 1989.

[9] R. Temam, "Navier-Stokes Equations - Theory and Numerical Analysis", North-Holland Publishing Company, Amsterdam-New York-Oxford, 1979.

[10] W. Xie, A sharp pointlibie bound for functions with $L^{2}$ laplacions and zero boundary values on arbitrary three-dimensional domains, Indiana University Mathematics Journal, 40 (1991), 1185-1192. 